\title{
A loss of hippocampal perineuronal nets produces deficits in dopamine system function: relevance to the positive symptoms of schizophrenia
}

\author{
A Shah and DJ Lodge
}

Deficits in parvalbumin containing interneurons are a consistent observation in animal models and schizophrenia patients. These neurons are surrounded by chondroitin sulfate proteoglycans, forming perineuronal nets, thought to support the high firing frequencies observed in these neurons. A loss of perineuronal nets has been observed post mortem in human schizophrenia patients, however, whether this contributes to the symptoms of schizophrenia is not known. Here we directly examine the effects of chondroitinase ABC degradation of ventral hippocampal (vHipp) perineuronal nets, and demonstrate that this results in an enhanced hippocampal activity and significant increase in dopamine neuron population activity. In addition, chondroitinase-treated rats display an augmented locomotor response to amphetamine, consistent with the enhanced response to psychomotor stimulants observed in schizophrenia patients. Taken together, these data demonstrate that a loss of vHipp perineuronal nets is sufficient, in and of itself, to induce aberrant hippocampal and dopamine system function consistent with that observed in rodent models and schizophrenia patients.

Translational Psychiatry (2013) 3, e215; doi:10.1038/tp.2012.145; published online 15 January 2013

\section{Introduction}

Schizophrenia is a disease affecting up to $1 \%$ of the population. ${ }^{1}$ Whereas the exact etiology of schizophrenia is not currently known, an alteration in GABAergic function is a consistent observation in both animal models as well as postmortem studies in schizophrenia patients. ${ }^{2-8}$ Specifically, post-mortem studies have demonstrated a reduction in cortical glutamic acid decarboxylase $;^{9-11}$ an upregulation of post-synaptic GABA-A receptors ${ }^{12,13}$ and a decrease in GABA uptake sites ${ }^{14-16}$ in schizophrenia patients. There is increasing evidence that deficits in GABAergic transmission are demonstrated to occur within specific classes of GABA ergic interneurons, with considerable attention being paid to those containing the calcium binding protein parvalbumin. ${ }^{3}$ Indeed, a decrease in parvalbumin expression has been reported in a diverse variety of animal models, including the methylazoxymethanol (MAM), ${ }^{5,17}$ neonatal ventral hippocampal lesion ${ }^{6}$ and phencyclidine models, ${ }^{7,8,18}$ and has been consistently demonstrated in schizophrenia patients, post mortem. ${ }^{10,19,20}$ Although not conclusively demonstrated, this decrease in parvalbumin expression is likely attributable to, or results in, aberrant interneuron function leading to attenuated perisomatic inhibition and an augmented pyramidal neuron activity. Indeed, we have previously demonstrated that MAMtreated rats (for review see Lodge et al. ${ }^{21}$ ) display a decrease in hippocampal parvalbumin positive neurons, deficits in evoked gamma oscillatory activity and an increase in hippocampal neuronal activity. ${ }^{5,22}$ This is consistent with imaging studies in schizophrenia patients that demonstrate increases in baseline hippocampal activity that are correlated with positive symptom severity. ${ }^{23-25}$

An increase in hippocampal activity, particularly in ventral subfields, is able to augment dopamine system function, known to be associated with the positive symptoms of schizophrenia. Specifically, activation of the vHipp has been demonstrated to increase the number of spontaneously active dopamine neurons in the ventral tegmental area (VTA) ${ }^{26-28}$ and this is correlated with increases in dopamine efflux in the nucleus accumbens. ${ }^{27,29,30}$ Furthermore, we have previously demonstrated that the pathological increase in dopamine system function, observed in the MAM-rodent model of schizophrenia, is directly attributable to aberrant ventral hippocampal activity. ${ }^{22}$ Thus, MAM-treated rats display an increase in dopamine neuron population activity and an augmented locomotor response to amphetamine administration, both of which can be normalized by tetrodotoxin inactivation of the vHipp. ${ }^{22}$ This has led us to posit that the positive symptoms of schizophrenia, known to be associated with augmented dopamine transmission, may be secondary to a pathological increase in ventral hippocampal activity. ${ }^{31}$ Furthermore, this increase in hippocampal activity may be attributable to a decreased function of perisomatic targeting, parvalbumin positive interneurons.

Parvalbumin containing interneurons in the cortex and hippocampus are encapsulated by chondroitin sulfate proteoglycans forming an extracellular matrix known as a perineuronal net (Figure 1). Although perineuronal nets were

Department of Pharmacology and Center for Biomedical Neuroscience, University of Texas Health Science Center, San Antonio, TX, USA

Correspondence: Dr DJ Lodge, Department of Pharmacology and Center for Biomedical Neuroscience, University of Texas Health Science Center, 7703 Floyd Curl Drive, MC 7764, San Antonio, TX 78229, USA.

E-mail: LodgeD@uthscsa.edu

Keywords: chondroitinase; dopamine; parvalbumin; perineuronal net; schizophrenia; ventral hippocampus

Received 6 September 2012; revised 16 November 2012; accepted 24 November 2012 


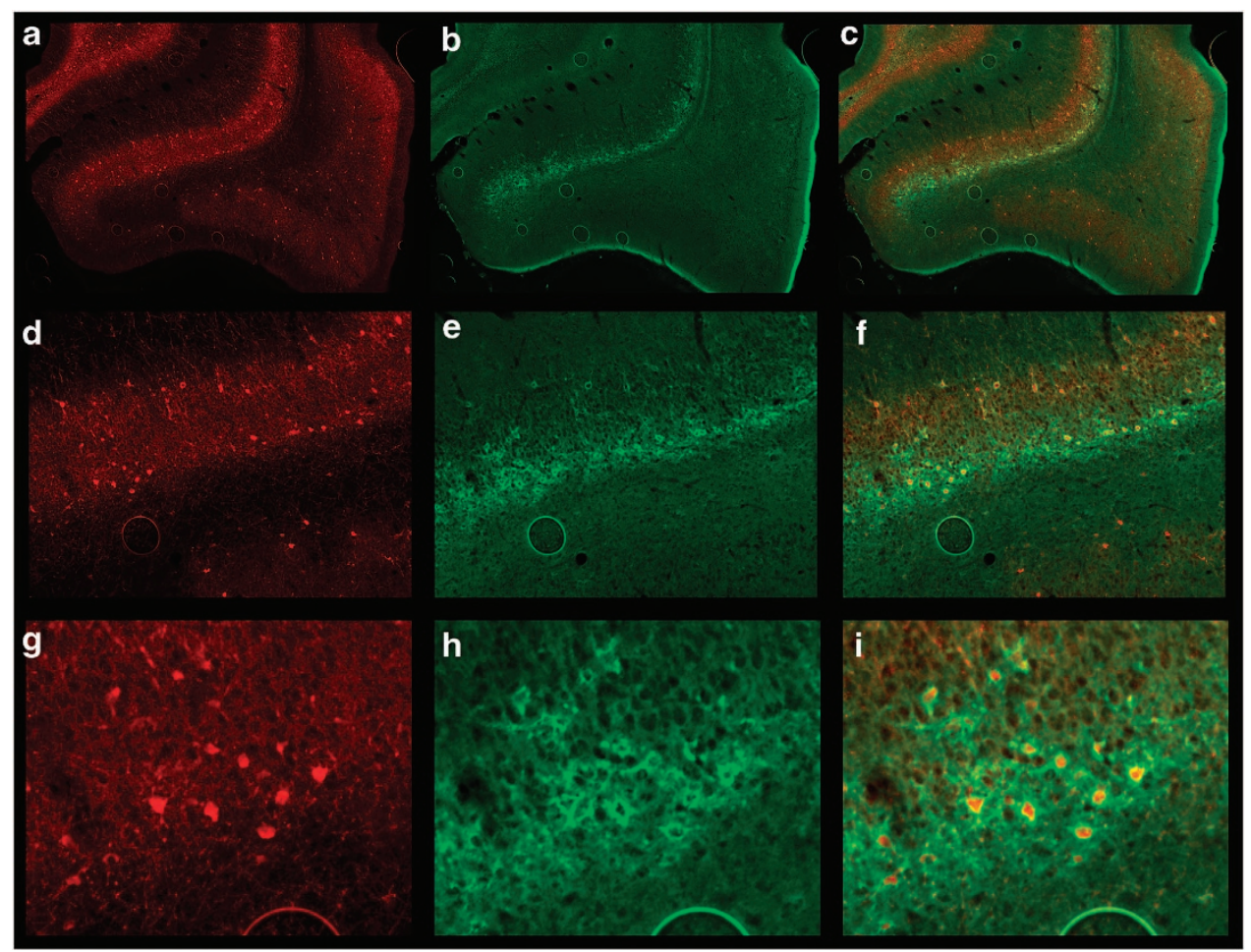

Figure 1 Perineuronal nets surround parvalbumin positive interneurons throughout the ventral hippocampus. Photomicrographs at increasing levels of magnification (from top to bottom) demonstrate the distribution of parvalbumin positive interneurons (red) and perineuronal nets (green) throughout the ventral regions of the hippocampus. The merged images (c, $\mathbf{f}$ and $\mathbf{i})$ demonstrate that a significant number of parvalbumin-containing neurons are enveloped by perineuronal nets, as determined by lectin labeling.

described by Golgi over a century ago, relatively little is known about their function. ${ }^{32}$ Recent work has suggested that perineuronal nets act to stabilize synapses, ${ }^{33}$ support synaptic plasticity ${ }^{34,35}$ and regulate the motility of extracellular ions. ${ }^{36,37}$ Given that perineuronal nets appear to envelop Kv3.1b expressing neurons, ${ }^{37}$ it has been suggested that these structures likely support the high firing frequencies of fast-spiking interneurons. Interestingly, a loss of perineuronal nets has been recently reported throughout the medial temporal lobe of postmortem schizophrenia patients. ${ }^{38,39}$ We suggest that a loss of perineuronal nets may contribute to the aberrant hippocampal activity and subsequent dopamine hyperfunction associated with the positive symptoms of schizophrenia. Here we examine whether a loss of vHipp perineuronal nets is sufficient, in and of itself, to produce physiological and behavioral deficits associated with the positive symptoms of schizophrenia.

\section{Materials and methods}

All experiments were performed in accordance with the guidelines outlined in the USPHS Guide for the Care and Use of Laboratory Animals, and were approved by the Institutional Animal Care and Use Committee of the University of Texas Health Science Center, San Antonio.

Animals. MAM acetate treatments were performed as described previously. ${ }^{40}$ In brief, timed pregnant female Sprague-Dawley rats were administered MAM $\left(25 \mathrm{mg} \mathrm{kg}^{-1}\right.$, i.p.) on GD17. Control rats received injections of saline $\left(1 \mathrm{ml} \mathrm{kg}^{-1}\right.$, i.p.). Male pups were weaned on day 21 and housed in groups of 2-3 with littermates until adulthood ( $>8$ weeks), at which time they were used for neurochemical studies.

Western blot. Adult MAM and saline-treated rats $(n=5$ per group: $300-450 \mathrm{~g}$ ) were anaesthetized with sodium pentobarbital (120 $\mathrm{mg} \mathrm{kg}^{-1}$, i.p.) and rapidly decapitated. The ventral hippocampus was dissected on ice and homogenized in disruption buffer $(50 \mathrm{~mm}$ Tris, $150 \mathrm{~mm} \mathrm{NaCl}, \mathrm{pH} 7.4$ ) containing a protease inhibitor cocktail (Sigma-Aldrich, St Louis, MO, USA: P8340). The perineuronal net fraction was isolated essentially as described previously. ${ }^{41}$ Specifically, homogenates were centrifuged at 14000 r.p.m. for $10 \mathrm{~min}$ and the pellet resuspended in disruption buffer containing $0.5 \%$ Triton $X-100$. The homogenate was centrifuged and the pellet resuspended in $200 \mu$ incubation buffer $(0.1 \mathrm{M}$ Tris, $0.03 \mathrm{M}$ sodium acetate, $\mathrm{pH} 8.0$ ) containing $0.1 \mathrm{U}$ of chondroitinase $A B C$ and incubated at $37^{\circ} \mathrm{C}$ for $2.5 \mathrm{~h}$. The homogenate was centrifuged and the supernatant analyzed as a purified perineuronal net fraction. Given that the initial washes are unlikely to release chondroitin sulfate proteoglycans from perineuronal nets, ${ }^{41}$ the proteins examined following chondroitinase $A B C$ digestion should be those associated with perineuronal nets; indeed we obtained dramatically different results when analyzing chondroitin sulfate proteoglycans from the cytosolic fractions (data not shown).

Proteins were separated on a 4-20\% gradient gel (Bio-Rad, Hercules, CA, USA) and transferred to polyvinyl difluoride 
membranes. Membranes were blocked and incubated with either mouse antineurocan 1:100 (DSHB, lowa City, IA, USA: 1F6-s), mouse antiphosphacan 1:100 (DSHB: 3F8-s), mouse antibrevican 1:1000 (BD Biosystems, San Jose, CA, USA: 610894) or mouse antiGAPDH 1:1000 (Abcam, Cambridge, MA, USA: ab9484), followed by HRP-antimouse 1:5000. Membranes were treated with an ECL substrate (Pierce, Rockford, IL, USA) and opposed to film prior to scanning and quantification with ImageJ.

Chondroitinase treatment. All survival surgical procedures were performed under general anesthesia in a semi-sterile environment. Briefly, male Sprague-Dawley rats (250-350 g) were pretreated with atropine $\left(0.1 \mathrm{mg} \mathrm{kg}^{-1}\right.$, i.p.), anesthetized with sodium pentobarbital $\left(60 \mathrm{mg} \mathrm{kg}^{-1}\right.$, i.p.) and placed in a stereotaxic apparatus. Chondroitinase ABC from Proteus vulgaris $\left(0.05 \mathrm{U}_{\mu} \mathrm{l}^{-1}\right)$ or penicillinase from Bacillus cereus $\left(0.05 \mathrm{U}_{\left.\mu \mathrm{l}^{-1}\right)}\right.$ were backfilled into a glass micropipette and pressure injected $(0.75 \mu \mathrm{l})$ bilaterally into the ventral hippocampus (A/P $-5.3, M / L \pm 5.2, D / V-7.5 \mathrm{~mm})$. The pipette was removed, the wound sutured, the rat removed from the stereotaxic frame and monitored closely until conscious. Rats were housed for at least a week before subsequent experiments.

Extracellular recordings. Extracellular recordings were performed as described previously. ${ }^{22}$ In brief, rats (300$450 \mathrm{~g}$ ) were anaesthetized with chloral hydrate (400 $\mathrm{mg} \mathrm{kg}^{-1}$, i.p.) and placed in a stereotaxic apparatus. Glass extracellular microelectrodes were lowered into the VTA $(n=14-16$ rats per group: A/P $-5.3, \mathrm{M} / \mathrm{L}+0.6 \mathrm{~mm}$ from bregma and from -6.5 to $-9.0 \mathrm{~mm}$ ventral of brain surface) or ventral hippocampus ( $n=7$ rats per group: A/P $-5.3, \mathrm{M} / \mathrm{L}+5.2 \mathrm{~mm}$ from bregma and from -5.0 to $-8.5 \mathrm{~mm}$ ventral of brain surface). Spontaneously active dopamine neurons were identified with open filter settings (low pass: $30 \mathrm{~Hz}$, high pass: $30 \mathrm{kHz}$ ) using previously established electrophysiological criteria, ${ }^{42}$ whereas putative pyramidal neurons were defined as those with firing frequencies $<2 \mathrm{~Hz}$ as reported previously. ${ }^{43,44}$

Locomotor analysis. Chondroitinase- or Penicillinase-treated rats ( $n=16-18$ per group: $300-450 \mathrm{~g}$ ) were placed in an open field arena (Med Associates, St Albans, VT, USA), where spontaneous locomotor activity in the $\mathrm{X}-\mathrm{Y}$ plane was determined for $45 \mathrm{~min}$ by beam breaks and recorded with Open Field Activity Software (Med Associates). Rats were then injected with amphetamine $\left(0.5 \mathrm{mg} \mathrm{kg}^{-1}\right.$, i.p.) and locomotor activity recorded for an additional $90 \mathrm{~min}$.

Histochemistry. Anesthetized rats were perfused transcardially with saline followed by formaldehyde and their brains removed, post fixed/cryopreserved for $24 \mathrm{~h}$. Coronal sections of the ventral hippocampus were used to detect expression of perineurnal nets. A subset of brains were also processed for parvalbumin immunohistochemistry. Briefly, sections were blocked ( $2 \%$ normal goat serum for $30 \mathrm{~min}$ ) followed by incubation with lectin from Wisteria floribunda $\left(4 \mu \mathrm{g} \mathrm{ml}^{-1}\right)$ and visualization by AlexaFluor 488 conjugated to streptavidin (1:500 for $1 \mathrm{~h}$ at room temperature). A subset of sections were also incubated with rabbit antiparvalbumin 1:1000 (Abcam: AB11427)) followed by antirabbit-AlexaFluor 594 (1:1000 for $1 \mathrm{~h}$ at room temperature). Sections of vHipp from each rat were visualized to confirm the loss of perineuronal nets in chondroitinase $\mathrm{ABC}$-treated rats.

Analysis. Electrophysiological analysis of dopamine and vHipp neuron activity was performed using commercially available computer software (LabChart v7.1). Locomotor activity was analyzed with Activity Monitor (Med Associates), whereas western blots films were scanned on an Epson Perfection V700 and optical density measured using ImageJ. Immunohistochemistry was examined on an Axio Lab.A1 Fluorescence Microscope and images taken with an attached AxioCam ICc1 digital camera. All data are represented as the mean \pm standard error of the mean (s.e.m.) unless otherwise stated. All statistics were calculated using SigmaPlot (Systat Software, Chicago, IL, USA).

Materials. MAM acetate was purchased from Midwest Research Institute (Kansas City, MO, USA). The monocloncal antibodies antineurocan (1F6-s) and antiphosphacan (3F8-s), developed by RU Margolis and RK Margolis, were obtained from the Developmental Studies Hybridoma Bank. The anti-brevican antibody (610894) was purchased from BD Biosciences, whereas the antiparvalbumin (ab11427) and antiGAPDH (ab9484) antibodies were purchased from ABCAM. The HRP-anti-mouse, AlexaFluor 596-conjugated anti-rabbit, AlexaFluor 488-conjugated to streptavidin and ProLong Gold antifade mountant were obtained from Invitrogen (Grand Island, NY, USA). The Laemmli Sample Buffer, Mini-Protean gels and polyvinyl difluoride membranes were from Bio-rad. Sodium pentobarbital, atropine, protease inhibitor cocktail (P8340), amphetamine, chondroitinase ABC from Proteus vulgaris, Penicillinase from Bacillus cereus and lectin from Wisteria floribunda were purchased from Sigma. All other chemicals and reagents were of either analytical or laboratory grade, and purchased from standard suppliers.

\section{Results}

Western blot. A loss of perineuronal nets has been described, post mortem, in schizophrenia patients. ${ }^{38,39}$ To examine whether this is also observed in rodent models of the disease, we examined the abundance of perineuronal net proteins throughout the vHipp of the MAM model of schizophrenia. MAM-treated rats demonstrated a significant decrease in the levels of the vHipp perineuronal net chondroitin sulfate proteoglycans, Brevican (SAL: $2.857 \pm 0.662$ c.f. MAM: $0.833 \pm 0.104 ; P<0.05$ Two-way ANOVA, Holm-Sidak $t=3.989, n=5$ rats per group in duplicate) and Phosphacan (SAL: $2.020 \pm 0.518$ c.f. MAM: $0.670 \pm 0.084 ; \quad P<0.05$ Two-way ANOVA, Holm-Sidak $t=2.661, n=5$ rats per group in duplicate), but not Neurocan (SAL: $1.102 \pm 0.195$ c.f. MAM: $0.665 \pm 0.101 ; P>0.05$ ), when compared to control, saline-treated rats (Figure 2).

In vivo electrophysiology. To confirm that a decrease in perineuronal nets results in aberrant vHipp activity, as 
reported in the MAM model, we performed in vivo recordings from putative vHipp pyramidal neurons. Specifically, chondroitinase-treated rats displayed a significantly greater average firing rate of putative pyramidal neurons when compared to rats receiving intra-vHipp penicillinase (Penn: $0.74 \pm 0.09 \mathrm{~Hz}$ c.f. Chase: $0.99 \pm 0.07 \mathrm{~Hz} ; \quad P<0.05$ Rank Sum Test, $n=41-63$ cells per group). These data suggest that a loss of perineuronal nets is sufficient to augment

a
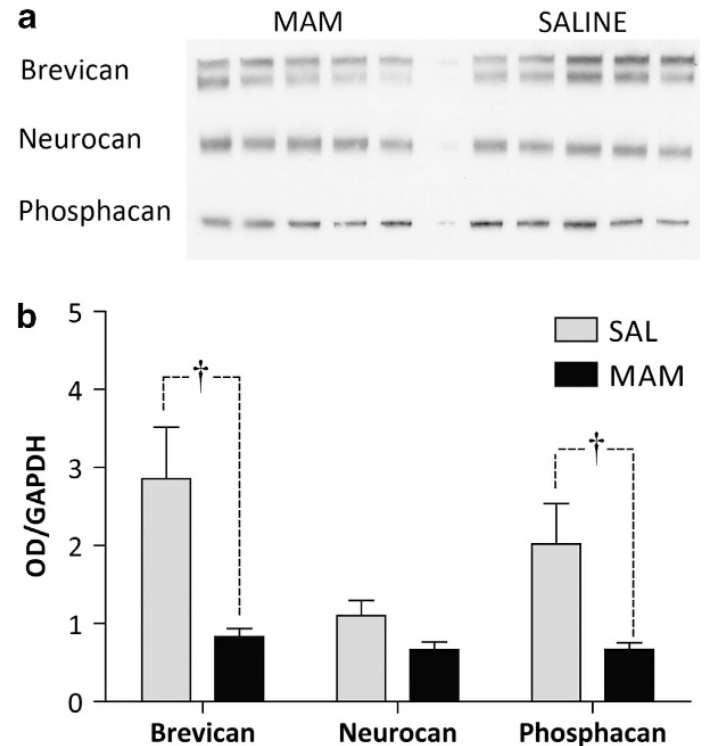

Figure 2 MAM-treated rats display decreases in the levels of the chondroitin sulfate proteoglycans associated with vHipp perineuronal nets. Representative films demonstrating the abundance of three chondroitin sulfate proteoglycans, brevican, neurocan and phosphacan are depicted in (a), whereas the quantitation of these data are presented in (b). " represents significant difference from saline-treated rats, Two-way ANOVA followed by Holm-Sidak post-hoc, $n=5$ rats per group in duplicate. hippocampal activity, presumably due to a decrease in fast-spiking interneuron function.

Given that an augmented vHipp activity has been demonstrated to increase dopamine system function, ${ }^{22,28,45}$ we recorded the activity of the population of spontaneously active dopamine neurons throughout the VTA of chondroitinase- and penicillinase-treated rats. Interestingly, the chondroitinaseinduced loss of hippocampal perineuronal nets resulted in a significant increase in the number of spontaneously activity dopamine neurons observed per electrode track (Penn: $0.92 \pm 0.11$ cells per track c.f. Chase: $1.73 \pm 0.13$ cells per track; $P<0.05$ Students $t$-test $t=-4.739 n=14-16$ rats per group) without significantly altering average firing rate or burst firing (Figure 3), similar to previous observations in the MAM model. ${ }^{22}$

Amphetamine-induced hyperlocomotion. We have previously demonstrated that increases in dopamine neuron activity, secondary to augmented vHipp activity, result in a behavioral hyperactivity to low doses of psychomotor stimulants. ${ }^{22,45}$ Here we demonstrate that rats treated with intra-vHipp chondroitinase $A B C$ display a significantly increased locomotor response to amphetamine when compared to penicillinase-treated rats (Two-way ANOVA; $\mathrm{F}_{\text {(Treatment) }}=40.845: n=16-18$ rats per group; Figure 4), demonstrating that a loss of hippocampal perineuronal nets results in behavioral alterations consistent with those observed in rodent models as well as in schizophrenia patients. ${ }^{46,47}$

\section{Discussion}

Here we demonstrate that a loss of ventral hippocampal perineuronal nets is sufficient to augment hippocampal activity and increase dopamine system function. Moreover, this is correlated with an enhanced behavioral response to
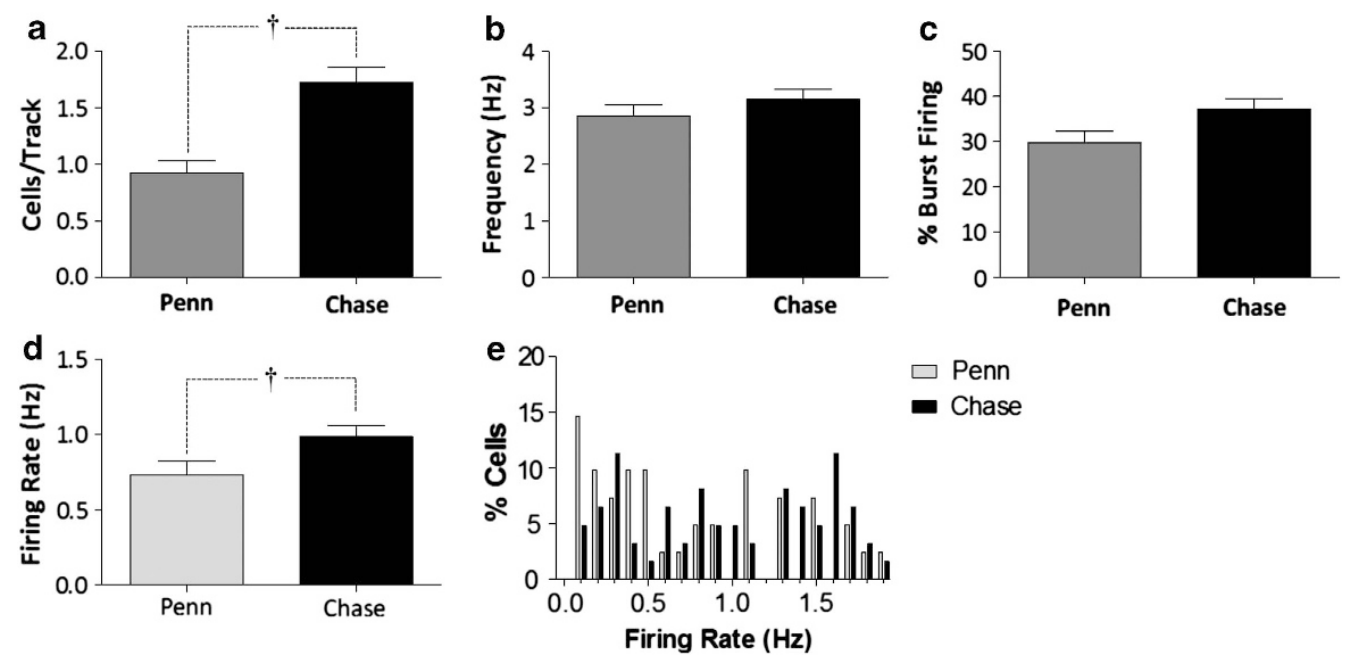

Figure 3 Enzymatic degradation of vHipp perineuronal nets augments dopamine and hippocampal activity. vHipp chondroitinase ABC treatment resulted in a selective increase in dopamine neuron population activity (a), with no significant effect on average firing rate (b), or burst firing (c) when compared to control, penicillinase-treated, rats. The degradation of perineuronal nets resulted in an increase in the average firing rate of purported pyramidal neurons throughout the vHipp (d and $\mathbf{e}$ ). ${ }^{\dagger}$ represents significant difference from penicillinase-treated rats, Students $t$-test (Rank Sum Test if data failed normality test), $n=14-16$ rats per group for $A, n=88-125$ cells per group for $\mathbf{b}$ and $\mathbf{c}$ and $n=41-63$ cells per group for ( $\mathbf{d}$ and $\mathbf{e})$. 


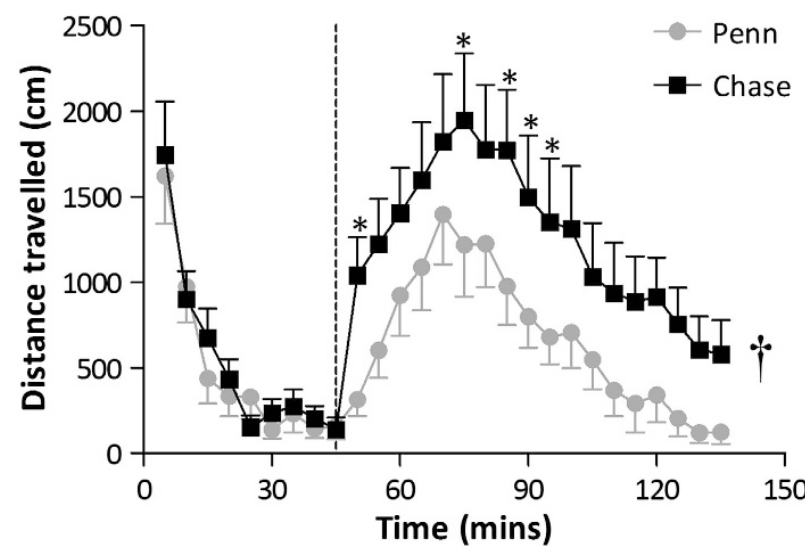

Figure 4 Enzymatic degradation of vHipp perineuronal nets results in an enhanced locomotor response to amphetamine. vHipp chondroitinase $A B C$ treatment did not appear to alter spontaneous locomotor activity in a novel environment; however, a significant increase in the locomotor response to amphetamine (administered at the dashed line) was observed in rats with perineuronal net degradation. †represents a mean effect of treatment, whereas *indicates significant difference between chondroitinase $A B C$ and penicillinase in pairwise multiple comparisons across time; Two-way ANOVA, followed by HolmSidak post-hoc, $n=16-18$ rats per group).

amphetamine, a marker of positive symptoms of schizophrenia. ${ }^{46,47}$ Given that a loss of perineuronal nets has been reported throughout the medial temporal lobe of post-mortem schizophrenia patients, ${ }^{38}$ we suggest that alterations in extracellular matrix proteins may have a significant role in the pathophysiology of schizophrenia.

As detailed above, a decreased expression of perineuronal nets has been reported throughout the temporal lobe ${ }^{38}$ of post-mortem schizophrenia patients. Here we demonstrate that the MAM-rodent model demonstrates significantly lower levels of the perineuronal net proteins, brevican and phosphacan, throughout the ventral hippocampus. It should be noted that MAM-treated rats also display reductions in parvalbumin-containing interneurons; ${ }^{5}$ thus, whether the decreased expression of perineuronal net proteins reflects a loss of parvalbumin neurons, or vice versa, is not currently known. Interestingly, these observed alterations in phosphacan expression are consistent with genetic association studies demonstrating that PTPRZ1, the gene encoding phosphacan, may influence the susceptibility for schizophrenia. ${ }^{48}$ Thus, the decreased expression of phosphacan further supports the validity of the MAM model, as it appears to mimic phosphacan abnormalities in schizophrenia patients, albeit via a different mechanism.

Given that we have previously demonstrated that MAMtreated rats display augmented vHipp activity that underlies the dopamine hyperfunction and behavioral hyperresponsivity to psychomotor stimulants, ${ }^{22}$ we investigated whether a decrease in vHipp perineuronal nets was sufficient to induce aberrant hippocampal and dopamine system function, thought to underlie the positive symptoms of the schizophrenia. Perineuronal nets throughout the vHipp were enzymatically degraded, in a region specific manner, by the administration of chondroitinase $\mathrm{ABC}$ from Proteus vulgaris. Chondroitinase $A B C$ degrades glycosaminoglycans, including chondroitin sulfate and hyaluronan, disrupting the structure of the perineuronal net. ${ }^{49}$ Indeed, qualitative examination of the vHipp following chondroitinase $A B C$ demonstrated a striking decrease in lectin staining to perineuronal nets (Figure 5). In contrast, the administration of penicillinase, from Bacillus cereus, did not appear to alter the distribution of perineuronal nets (Figure 5), and was therefore used as a control.

It has been suggested that perineuronal nets support the high firing frequencies of fast-spiking, parvalbumin-containing interneurons. Given that these are perisomatic targeting interneurons, a loss of function would be anticipated to increase pyramidal activity. Indeed, here we demonstrate a significantly greater firing rate of putative pyramidal neurons throughout the ventral hippocampus of chondroitinasetreated rats, when compared to penicillinase-treated controls. Interestingly, this increase in hippocampal function is qualitatively similar to that observed in the MAM model, ${ }^{22}$ and is consistent with human imaging data demonstrating augmented hippocampal activity in schizophrenia patients. ${ }^{23-25}$ It should be noted that hippocampal cultures treated in vitro with chondroitinase $\mathrm{ABC}$ do not display altered electrophysiological parameters including resistance, capacitance, resting membrane potential, nor were the amplitude or half width of action potentials of interneurons altered. ${ }^{50}$ Thus, the results observed in the current study likely reflect alterations in interneuron function that are either a consequence of the treatment regimen (that is, one injection with animals tested $>1$ week later c.f. $48 \mathrm{~h}$ of direct exposure in vitro) or, alternatively, may require the normal patterns of afferent input observed in the intact brain. Indeed, the genetic deletion of tenascin, an integral component of the perineuronal net, results in a decrease in perisomatic inhibition and enhanced excitatory synaptic transmission of pyramidal neurons of the hippocampus, ${ }^{51}$ consistent with the data presented here.

We have previously demonstrated that the hippocampus can regulate dopamine system function via a multi-synaptic pathway including the nucleus accumbens and ventral pallidum (for review see Lodge et al. ${ }^{31}$ ). Moreover, we posit that the aberrant vHipp activity may actually be a primary pathology underlying the positive symptoms of schizophrenia. For this reason, we examined whether the chondroitinaseinduced increase in hippocampal activity was sufficient to augment dopamine neuron activity in the VTA. Indeed, chondroitinase-treated rats displayed a significantly greater number of spontaneously active VTA dopamine neurons when compared to penicillinase-treated controls. The number of dopamine neurons firing spontaneously is thought to reflect the gain of the dopamine system whereby the functionally relevant, phasic signal can be amplified or dampened based on environmental context and novelty. ${ }^{28}$ Thus, a sustained increase in the number of spontaneously active dopamine neurons would artificially ascribe high salience to events that are not typically salient, and may contribute to the positive symptoms of schizophrenia. ${ }^{31}$ An increase in dopamine neuron population activity has been previously suggested to underlie the augmented response to psychomotor stimulants observed in animal models, ${ }^{22,40,52}$ as well as schizophrenia patients. ${ }^{46,47}$ This can be examined in rodent models by investigating the locomotor response to a low dose of amphetamine. ${ }^{22,45,53}$ Indeed, we now demonstrate that 

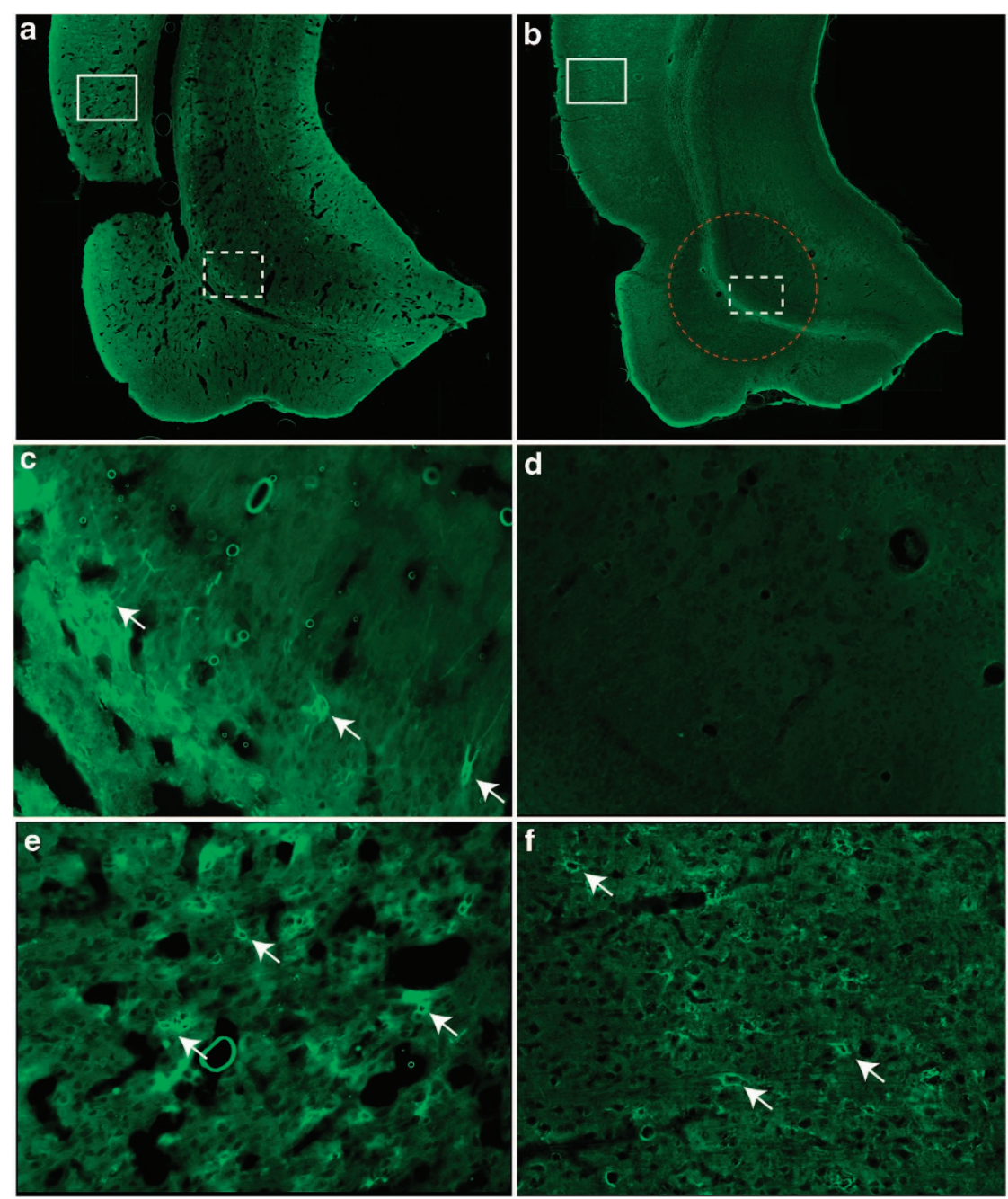

Figure 5 Intra-vHipp administration of chondroitinase $A B C(\mathbf{b}, \mathbf{d}$ and $\mathbf{f})$, but not penicillinase (a,c and $\mathbf{e})$, results in a significant decrease in lectin staining to perineuronal nets throughout the vHipp. Photomicrographs demonstrate the extent of perineuronal net degradation, indicated by the red circle in (b). Chondroitinase $\mathrm{ABC}$ resulted in a region specific reduction of perineuronal nets observed in the vHipp (dashed box in $\mathbf{a}$ and $\mathbf{b}$-magnified in $\mathbf{c}$ and $\mathbf{d}$ ) but not in more dorsal regions of the cortex (solid box in $\mathbf{a}$ and $\mathbf{b}$ - magnified in $\mathbf{e}$ and $\mathbf{f}$ ). Penicillinase-treated rats display numerous perineuronal nets (indicated by arrows) throughout both the vHipp (c) and cortex (e).

chondroitinase-treated rats display a significantly greater locomotor response to amphetamine administration when compared to penicillinase-treated rats. Taken together, these data demonstrate that a loss of hippocampal perineuronal nets is sufficient, in and of itself, to augment hippocampal activity and induce the dopamine system hyperfunction purported to underlie the positive symptoms of schizophrenia.

\section{Conflict of Interest}

The authors declare no conflict of interest.

Acknowledgements. We would like to thank Amber Asher for her technical assistance with the immunohistochemistry. This work was supported by the NIH (R01: MH090067) and a NARSAD award from the Maltz Family Foundation. The monocloncal antibodies antineurocan (1F6-s) and antiphosphacan (3F8-s), developed by RU Margolis and RK Margolis, were obtained from the Developmental
Studies Hybridoma Bank developed under the auspices of the NICHD and maintained by the University of lowa Department of Biology, lowa City, IA, USA.

1. Stilo SA, Murray RM. The epidemiology of schizophrenia: replacing dogma with knowledge. Dialogues Clin Neurosci 2010; 12: 305-315.

2. Benes FM, Lim B, Matzilevich D, Walsh JP, Subburaju S, Minns M. Regulation of the GABA cell phenotype in hippocampus of schizophrenics and bipolars. Proc Nat Acad Sci 2007; 104: 10164-10169.

3. Lewis DA, Hashimoto T, Volk DW. Cortical inhibitory neurons and schizophrenia. Nat Rev Neurosci 2005; 6: 312-324.

4. Reynolds GP, Beasley CL, Zhang ZJ. Understanding the neurotransmitter pathology of schizophrenia: selective deficits of subtypes of cortical GABAergic neurons. J Neural Transmission 2002; 109: 881-889.

5. Lodge DJ, Behrens MM, Grace AA. A loss of parvalbumin-containing interneurons is associated with diminished oscillatory activity in an animal model of schizophrenia. $J$ Neurosci 2009; 29: 2344-2354.

6. Franois J, Ferrandon A, Koning E, Angst MJ, Sandner G, Nehlig A. Selective reorganization of GABAergic transmission in neonatal ventral hippocampal-lesioned rats. Int J Neuropsychopharmacol 2009; 12: 1097-1110.

7. Amitai N, Kuczenski R, Behrens MM, Markou A. Repeated phencyclidine administration alters glutamate release and decreases GABA markers in the prefrontal cortex of rats. Neuropharmacology 2012; 62: 1422-1431. 
8. Wang CZ, Yang SF, Xia Y, Johnson KM. Postnatal phencyclidine administration selectively reduces adult cortical parvalbumin-containing interneurons. Neuropsychopharmacology 2008; 33: 2442-2455.

9. Akbarian S, Kim JJ, Potkin SG, Hagman JO, Tafazzoli A, Bunney Jr WE et al. Gene expression for glutamic acid decarboxylase is reduced without loss of neurons in prefronta cortex of schizophrenics. Arch Gen Psychiatry 1995; 52: 258-278.

10. Hashimoto T, Volk DW, Eggan SM, Mirnics K, Pierri JN, Sun Z et al. Gene expression deficits in a subclass of GABA neurons in the prefrontal cortex of subjects with schizophrenia. J Neurosci 2003; 23: 6315-6326.

11. Volk DW, Austin MC, Pierri JN, Sampson AR, Lewis DA. Decreased glutamic acid decarboxylase67 messenger RNA expression in a subset of prefrontal cortical? aminobutyric acid neurons in subjects with schizophrenia. Arch Gen Psychiatry 2000; 57: 237-245

12. Benes FM, Vincent SL, Alsterberg G, Bird ED, SanGiovanni JP. Increased GABA(A) receptor binding in superficial layers of cingulate cortex in schizophrenics. J Neurosci 1992 12: 924-929.

13. Benes FM, Vincent SL, Marie A, Khan Y. Up-regulation of GABA(A) receptor binding on neurons of the prefrontal cortex in schizophrenic subjects. Neuroscience 1996; 75: 1021-1031.

14. Reynolds GP, Czudek C, Andrews HB. Deficit and hemispheric asymmetry of GABA uptake sites in the hippocampus in schizophrenia. Biol Psychiatry 1990; 27: 1038-1044.

15. Simpson MDC, Slater P, Deakin JFW, Royston MC, Skan WJ. Reduced GABA uptake sites in the temporal lobe in schizophrenia. Neurosci Lett 1989; 107: 211-215.

16. Simpson MDC, Slater $P$, Royston MC, Deakin JFW. Regionally selective deficits in uptake sites for glutamate and gamma-aminobutyric acid in the basal ganglia in schizophrenia Psychiatry Res 1992; 42: 273-282.

17. Penschuck S, Flagstad P, Didriksen M, Leist M, Michael-Titus AT. Decrease in parvalbumin-expressing neurons in the hippocampus and increased phencyclidineinduced locomotor activity in the rat methylazoxymethanol (MAM) model of schizophrenia. Eur J Neurosci 2006; 23: 279-284.

18. Jenkins TA, Harte MK, Reynolds GP. Effect of subchronic phencyclidine administration on sucrose preference and hippocampal parvalbumin immunoreactivity in the rat. Neurosci Lett 2010; 471: 144-147.

19. Beasley CL, Zhang ZJ, Patten I, Reynolds GP. Selective deficits in prefrontal cortical GABAergic neurons in schizophrenia defined by the presence of calcium-binding proteins. Biol Psychiatry 2002; 52: 708-715.

20. Knable MB, Barci BM, Webster MJ, Meador-Woodruff J, Torrey EF. Molecular abnormalities of the hippocampus in severe psychiatric illness: postmortem findings from the Stanley Neuropathology Consortium. Mol Psychiatry 2004; 9: 609-620.

21. Lodge DJ, Grace AA. Gestational methylazoxymethanol acetate administration: a developmental disruption model of schizophrenia. Behav Brain Res 2009; 204: 306-312.

22. Lodge DJ, Grace AA. Aberrant hippocampal activity underlies the dopamine dysregulation in an animal model of schizophrenia. J Neurosci 2007; 27: 11424-11430.

23. Heckers S, Rauch SL, Goff D, Savage CR, Schacter DL, Fischman AJ et al. Impaired recruitment of the hippocampus during conscious recollection in schizophrenia. Nat Neurosci 1998; 1: 318-323.

24. Lahti AC, Weiler MA, Holcomb HH, Tamminga CA, Carpenter WT, McMahon R Correlations between rCBF and symptoms in two independent cohorts of drug-free patients with schizophrenia. Neuropsychopharmacology 2006; 31: 221-230.

25. Nordahl TE, Kusubov N, Carter C, Salamat S, Cummings AM, O'Shora-Celaya L et al. Temporal lobe metabolic differences in medication-free outpatients with schizophrenia via the PET-600. Neuropsychopharmacology 1996; 15: 541-554.

26. Floresco SB, Todd CL, Grace AA. Glutamatergic afferents from the hippocampus to the nucleus accumbens regulate activity of ventral tegmental area dopamine neurons. J Neurosci 2001; 21: 4915-4922.

27. Floresco SB, West AR, Ash B, Moore H, Grace AA. Afferent modulation of dopamine neuron firing differentially regulates tonic and phasic dopamine transmission. Nat Neurosci 2003; 6: 968-973.

28. Lodge DJ, Grace AA. The hippocampus modulates dopamine neuron responsivity by regulating the intensity of phasic neuron activation. Neuropsychopharmacology 2006; 31 1356-1361.

29. Blaha CD, Yang CR, Floresco SB, Barr AM, Phillips AG. Stimulation of the ventra subiculum of the hippocampus evokes glutamate receptor-mediated changes in dopamine efflux in the rat nucleus accumbens. European J Neurosci 1997; 9: 902-911.

30. Taepavarapruk $P$, Floresco SB, Phillips AG. Hyperlocomotion and increased dopamine efflux in the rat nucleus accumbens evoked by electrical stimulation of the ventra subiculum: role of ionotropic glutamate and dopamine D1 receptors. Psychopharmacology (Berl) 2000; 151: 242-251.

31. Lodge DJ, Grace AA. Hippocampal dysregulation of dopamine system function and the pathophysiology of schizophrenia. Trends Pharmacological Sci 2011; 32: 507-513.

32. Celio MR, Spreafico R, De Biasi S, Vitellaro-Zuccarello L. Perineuronal nets: past and present. Trends Neurosci 1998; 21: 510-515.
33. Berardi N, Pizzorusso T, Maffei L. Extracellular matrix and visual cortical plasticity: freeing the synapse. Neuron 2004; 44: 905-908.

34. Bukalo O, Schachner M, Dityatev A. Modification of extracellular matrix by enzymatic removal of chondroitin sulfate and by lack of tenascin-R differentially affects several forms of synaptic plasticity in the hippocampus. Neuroscience 2001; 104: 359-369.

35. Brakebusch C, Seidenbecher Cl, Asztely F, Rauch U, Matthies H, Meyer H et al. Brevicandeficient mice display impaired hippocampal CA1 long-term potentiation but show no obvious deficits in learning and memory. Mol Cell Biol 2002; 22: 7417-7427.

36. Bruckner G, Brauer K, Hartig W, Wolff JR, Rickmann MJ, Derouiche A et al. Perineuronal nets provide a polyanionic, glia-associated form of microenvironment around certain neurons in many parts of the rat brain. Glia 1993; 8: 183-200.

37. Hartig W, Derouiche A, Welt K, Brauer K, Grosche J, Mader M et al. Cortical neurons immunoreactive for the potassium channel Kv3.1b subunit are predominantly surrounded by perineuronal nets presumed as a buffering system for cations. Brain Res 1999; 842: 15-29.

38. Pantazopoulos H, Woo TUW, Lim MP, Lange N, Berretta S. Extracellular matrix-glial abnormalities in the amygdala and entorhinal cortex of subjects diagnosed with schizophrenia. Arch Gen Psychiatry 2010; 67: 155-166.

39. Berretta S. Extracellular matrix abnormalities in schizophrenia. Neuropharmacology 2012; 62: 1584-1597.

40. Moore H, Jentsch JD, Ghajarnia M, Geyer MA, Grace AA. A neurobehavioral systems analysis of adult rats exposed to methylazoxymethanol acetate on E17: implications for the neuropathology of schizophrenia. Biol Psychiatry 2006; 60: 253-264.

41. Deepa SS, Carulli D, Galtrey C, Rhodes K, Fukuda J, Mikami T et al. Composition of perineuronal net extracellular matrix in rat brain: a different disaccharide composition for the net-associated proteoglycans. J Biol Chem 2006; 281: 17789-17800.

42. Grace AA, Bunney BS. Intracellular and extracellular electrophysiology of nigral dopaminergic neurons-1. identification and characterization. Neuroscience 1983; 10: 301-315.

43. Van Der Meer MAA, Redish AD. Theta phase precession in rat ventral striatum links place and reward information. $J$ Neurosci $2011 ; 31: 2843-2854$.

44. Ranck Jr JB. Studies on single neurons in dorsal hippocampal formation and septum in unrestrained rats. I. Behavioral correlates and firing repertoires. Experimental Neurol 1973; 41: $461-531$

45. Lodge DJ, Grace AA. Amphetamine activation of hippocampal drive of mesolimbic dopamine neurons: a mechanism of behavioral sensitization. I Neurosci 2008; 28: 7876-7882.

46. Breier A, Su TP, Saunders R, Carson RE, Kolachana BS, de Bartolomeis A et al. Schizophrenia is associated with elevated amphetamine-induced synaptic dopamine concentrations: evidence from a novel positron emission tomography method. Proc Nat Acad Sci 1997; 94: 2569-2574.

47. Laruelle M, Abi-Dargham A, Van Dyck CH, Gil R, D'Souza CD, Erdos J et al. Single photon emission computerized tomography imaging of amphetamine-induced dopamine release in drug-free schizophrenic subjects. Proc Nat Acad Sci 1996; 93: 9235-9240.

48. Buxbaum JD, Georgieva L, Young JJ, Plescia C, Kajiwara Y, Jiang Y et al. Molecular dissection of NRG1-ERBB4 signaling implicates PTPRZ1 as a potential schizophrenia susceptibility gene. Mol Psychiatry 2008; 13: 162-172.

49. Galtrey CM, Fawcett JW. The role of chondroitin sulfate proteoglycans in regeneration and plasticity in the central nervous system. Brain Res Rev 2007; 54: 1-18.

50. Dityatev A, Bruckner G, Dityateva G, Grosche J, Kleene R, Schachner M. Activitydependent formation and functions of chondroitin sulfate-rich extracellular matrix of perineuronal nets. Developmental Neurobiol 2007; 67: 570-588.

51. Saghatelyan AK, Dityatev A, Schmidt S, Schuster T, Bartsch U, Schachner M. Reduced perisomatic inhibition, increased excitatory transmission, and impaired long-term potentiation in mice deficient for the extracellular matrix glycoprotein tenascin-R. Mol Cell Neurosci 2001; 17: 226-240.

52. Flagstad P, Mork A, Glenthoj BY, van Beek J, Michael-Titus AT, Didriksen M. Disruption of neurogenesis on gestational day 17 in the rat causes behavioral changes relevant to positive and negative schizophrenia symptoms and alters amphetamine-induced dopamine release in nucleus accumbens. Neuropsychopharmacology 2004; 29 : 2052-2064.

53. White IM, Whitaker C, White W. Amphetamine-induced hyperlocomotion in rats: hippocampal modulation of the nucleus accumbens. Hippocampus 2006; 16: 596-603.

Translational Psychiatry is an open-access journal published by Nature Publishing Group. This work is licensed under the Creative Commons Attribution-NonCommercial-No Derivative Works 3.0 Unported License. To view a copy of this license, visit http://creativecommons.org/licenses/by-nc-nd/3.0/ 